POS $\quad$ PROCEEDINGS

\title{
Observables for Generalized TMDs of Quarks
}

\section{Shohini Bhattacharya}

Department of Physics, SERC, Temple University, Philadelphia, PA 19122, USA

E-mail: tug23108@temple.edu

\section{Andreas Metz*}

Department of Physics, SERC, Temple University, Philadelphia, PA 19122, USA

E-mail: metza@temple.edu

\section{Jian Zhou}

School of Physics and Key Laboratory of Particle Physics and Particle Irradiation (MOE), Shandong University, Jinan, Shandong 250100, China

E-mail: jzhou@sdu.edu. cn

Generalized TMDs (GTMDs) of hadrons are the most general two-parton correlation functions. Upon certain projections, several GTMDs reduce to generalized parton distributions (GPDs) and transverse momentum dependent parton distributions (TMDs), respectively. Therefore, GTMDs can be considered as partonic "mother functions". Moreover, two of the GTMDs play an important role in the nucleon spin structure. We show that, by means of the exclusive double Drell-Yan process, GTMDs for quarks can in principle be measured. This is the first known process which is sensitive to these objects. Specific GTMDs can be addressed via suitable polarization observables. We also identify other processes that are directly sensitive to GTMDs.

QCD Evolution 2017

22-26 May, 2017

Jefferson Lab, Newport News, VA - USA

${ }^{*}$ Speaker. 


\section{Introduction}

Multi-dimensional imaging of strongly interacting systems is currently a very active research area. The key quantities of this field are new types of parton distribution functions (PDFs) which are extensions of the one-dimensional PDFs that became textbook material - generalized parton distributions (GPDs) and transverse momentum dependent parton distributions (TMDs), which provide multi-dimensional images of hadrons in position space and momentum space, respectively. Studying GPDs and TMDs is a core mission at several particle accelerator facilities worldwide and, in particular, at a potential future electron-ion collider (EIC) in the United States [1,2].

In this context, GTMDs [3, 4, 5] have attracted enormous interest over the past few years. Since several GTMDs reduce to GPDs and TMDs in certain kinematical limits, they are often denoted as "mother distributions." The Fourier transform of GTMDs are Wigner functions [6, 7], the quantum-mechanical counterpart of classical phase-space distributions. Partonic Wigner functions contain information on the five-dimensional parton structure - the (average) longitudinal and transverse momentum as well as transverse position of partons inside a hadron [8]. Two of the GTMDs $-F_{1,4}$ and $G_{1,1}$ in the notation of [4] — play a crucial role in relation to the spin structure of the nucleon. Both functions describe the strength of spin-orbit interactions that are similar to spin-orbit interactions in atomic systems like hydrogen [9, 10, 11]. In particular, there is a direct relation between $F_{1,4}$ and the orbital angular momentum (OAM) of partons inside a longitudinally polarized nucleon $[9,12,13]$. It is remarkable that the same relation between $F_{1,4}$ and the quark OAM holds for both commonly used OAM definitions - the (canonical) one by Jaffe and Manohar $\left(L_{\mathrm{JM}}\right)$ [14], and the one by $\mathrm{Ji}\left(L_{\mathrm{Ji}}\right)$ [15]. This representation of OAM also allows for an intuitive interpretation of the difference between $L_{\mathrm{JM}}$ and $L_{\mathrm{Ji}}$ [16]. Moreover, it gives access to the so far elusive $L_{\mathrm{JM}}$ in quantum chromodynamics (QCD) on the lattice $[12,17,18]$.

While a number of model calculations of GTMDs is available by now, for many years it was unknown how GTMDs can be measured. Only recently it was shown that GTMDs of gluons can, in principle, be accessed via diffractive di-jet production in deep-inelastic lepton-nucleon and leptonnucleus scattering [19, 20, 21], as well as virtual photon-nucleus quasi-elastic scattering [22]. Some numerical studies of gluon GTMDs at small $x$, based on a saturation model, were performed in Refs. [23, 22]. Not long ago, it was also pointed out that gluon GTMDs can be studied in protonnucleus collisions [24]. With the exception of [21], the papers on observables for GTMDs deal with the small- $x$ region of parton saturation.

In a recent paper [25] we identified, for the first time, a physical process which gives access to quark GTMDs. Specifically, we showed how GTMDs enter the exclusive pion-nucleon double Drell-Yan process, $\pi N \rightarrow\left(\ell_{1}^{-} \ell_{1}^{+}\right)\left(\ell_{2}^{-} \ell_{2}^{+}\right) N^{\prime}$, where one detects two di-lepton pairs plus a nucleon. To this end, we performed a leading-order (LO) analysis in perturbative QCD. Our main focus was on the GTMDs $F_{1,4}$ and $G_{1,1}$, which can be measured through suitable polarization observables. We also argued that other quark GTMDs could be systematically studied in the same process. Here we report on our work in [25].

\section{Generalized TMDs}

Let us first recall the definition of quark GTMDs for a nucleon [3, 4]. GTMDs parameterize 
the off-forward transverse momentum dependent quark-quark correlator

$$
W_{\lambda, \lambda^{\prime}}^{q[\Gamma]}\left(P, \Delta, x, \vec{k}_{\perp}\right)=\left.\int \frac{d z^{-} d^{2} \vec{z}_{\perp}}{2(2 \pi)^{3}} e^{i k \cdot z}\left\langle p^{\prime}, \lambda^{\prime}\left|\bar{q}\left(-\frac{z}{2}\right) \Gamma \mathscr{W}\left(-\frac{z}{2}, \frac{z}{2}\right) q\left(\frac{z}{2}\right)\right| p, \lambda\right\rangle\right|_{z^{+}=0},
$$

where $q$ indicates the quark flavor and $\Gamma$ a generic gamma matrix. The 4-momenta and the helicities of the incoming (outgoing) nucleon are denoted by $p\left(p^{\prime}\right)$ and $\lambda\left(\lambda^{\prime}\right)$, respectively. We also use the definitions $P=\left(p+p^{\prime}\right) / 2$ and $\Delta=p^{\prime}-p$. The two quark fields of the operator in (2.1) are separated along the light-cone minus direction $z^{-}$and the transverse direction $\vec{z}_{\perp}$. (We define the light-cone components of a generic 4-vector $a=\left(a^{0}, a^{1}, a^{2}, a^{3}\right)$ through $a^{ \pm}=\left(a^{0} \pm a^{3}\right) / \sqrt{2}$ and $\vec{a}_{\perp}=\left(a^{1}, a^{2}\right)$.) The Wilson line $\mathscr{W}$ makes the bi-local operator color gauge invariant. The average longitudinal and transverse quark momenta are given by $x$ and $\vec{k}_{\perp}$, respectively. Strictly speaking, some modification of the definition in (2.1) is needed in order to avoid the infamous light-cone singularities. One way of regulating such singularities is to invoke the scheme proposed in Ref. [26], which is widely used in the TMD case. More information on this point can be found, for instance, in [27].

For pion-nucleon Drell-Yan one needs the parametrization of (2.1) in terms of GTMDs for $\Gamma=\gamma^{+}, \gamma^{+} \gamma_{5}$. In the notation of [4] one has [25]

$$
\begin{aligned}
W_{\lambda, \lambda^{\prime}}^{q\left[\gamma^{+}\right]}=\frac{1}{2 M} \bar{u}\left(p^{\prime}, \lambda^{\prime}\right)[ & \left.F_{1,1}^{q}+\frac{i \sigma^{i+} k_{\perp}^{i}}{P^{+}} F_{1,2}^{q}+\frac{i \sigma^{i+} \Delta_{\perp}^{i}}{P^{+}} F_{1,3}^{q}+\frac{i \sigma^{i j} k_{\perp}^{i} \Delta_{\perp}^{j}}{M^{2}} F_{1,4}^{q}\right] u(p, \lambda) \\
=\frac{1}{M \sqrt{1-\xi^{2}}}\{[ & \left.M \delta_{\lambda, \lambda^{\prime}}-\frac{1}{2}\left(\lambda \Delta_{\perp}^{1}+i \Delta_{\perp}^{2}\right) \delta_{\lambda,-\lambda^{\prime}}\right] F_{1,1}^{q}+\left(1-\xi^{2}\right)\left(\lambda k_{\perp}^{1}+i k_{\perp}^{2}\right) \delta_{\lambda,-\lambda^{\prime}} F_{1,2}^{q} \\
& +\left(1-\xi^{2}\right)\left(\lambda \Delta_{\perp}^{1}+i \Delta_{\perp}^{2}\right) \delta_{\lambda,-\lambda^{\prime}} F_{1,3}^{q} \\
& \left.+\frac{i \varepsilon_{\perp}^{i j} k_{\perp}^{i} \Delta_{\perp}^{j}}{M^{2}}\left[\lambda M \delta_{\lambda, \lambda^{\prime}}-\frac{\xi}{2}\left(\Delta_{\perp}^{1}+i \lambda \Delta_{\perp}^{2}\right) \delta_{\lambda,-\lambda^{\prime}}\right] F_{1,4}^{q}\right\}
\end{aligned}
$$

where the corresponding equation for $W_{\lambda, \lambda^{\prime}}^{q\left[\gamma^{+} \gamma_{5}\right]}$ can be found in [25]. In order to evaluate the first line of Eq. (2.2) we considered $u(p, \lambda)$ and $u\left(p^{\prime}, \lambda^{\prime}\right)$ as light-cone helicity spinors [28, 29]. Note that $M$ is the nucleon mass, and $\xi=\left(p^{+}-p^{\prime+}\right) /\left(p^{+}+p^{\prime+}\right)=-\Delta^{+} /\left(2 P^{+}\right)$characterizes the longitudinal momentum transfer to the nucleon. We also use $\sigma^{\mu v}=i\left[\gamma^{\mu}, \gamma^{v}\right] / 2$, and $\varepsilon_{\perp}^{i j}=\varepsilon^{-+i j}$ with the convention $\varepsilon^{0123}=1$. The kinematical arguments on the 1.h.s. of (2.2) are suppressed. For a generic GTMD one has $X=X\left(x, \xi, \vec{k}_{\perp}, \vec{\Delta}_{\perp}\right)$, where the dependence on $\vec{k}_{\perp}$ and $\vec{\Delta}_{\perp}$ is through the scalar products which can be formed by these vectors. We also recall that, in general, GTMDs are complex-valued functions [3, 4]. As discussed above our focus is on the GTMDs $F_{1,4}$ and $G_{1,1}$. The real part of the GTMDs $F_{1,1}$ and $G_{1,4}$ has a close connection to the distribution of unpolarized quarks in an unpolarized nucleon and the distribution of longitudinally polarized quarks in a longitudinally polarized nucleon, respectively $[4,9]$. Since these distributions are large we also consider observables which are sensitive to their interference with $F_{1,4}$ and $G_{1,1}$. Below we will concentrate on the helicity-conserving terms in (2.2) (and the corresponding equation for $W_{\lambda, \lambda^{\prime}}^{q\left[\gamma^{+} \gamma_{5}\right]}$ ) that are proportional to $\delta_{\lambda, \lambda^{\prime}}$.

The cross section for the double Drell-Yan process is also sensitive to the matrix element

$$
\Phi^{q}\left(x, \vec{k}_{\perp}^{2}\right)=\left.\int \frac{d z^{+} d^{2} \vec{z}_{\perp}}{2(2 \pi)^{3}} e^{i(k-p / 2) \cdot z}\left\langle 0\left|\bar{q}\left(-\frac{z}{2}\right) \gamma^{-} \gamma_{5} \mathscr{W}\left(-\frac{z}{2}, \frac{z}{2}\right) q\left(\frac{z}{2}\right)\right| \pi(p)\right\rangle\right|_{z^{-}=0}
$$




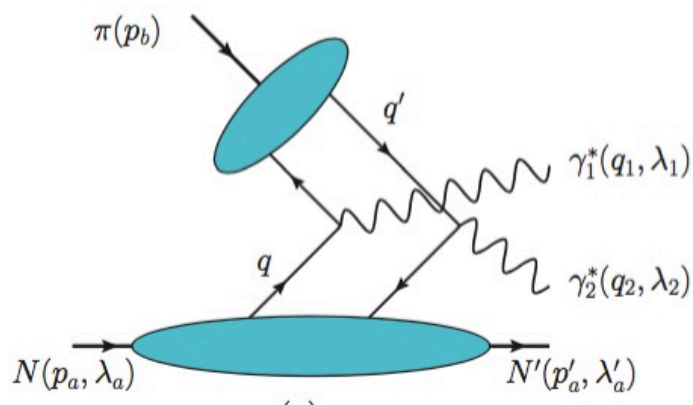

(a)

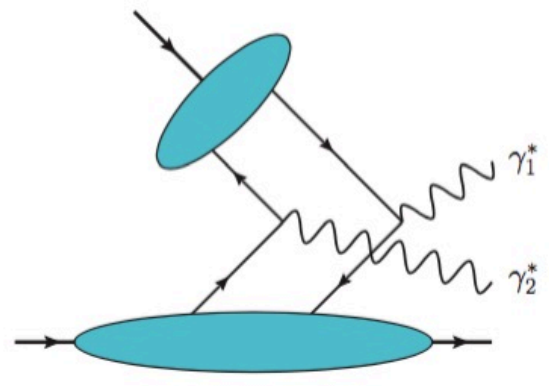

(b)

Figure 1: LO diagrams for the exclusive double Drell-Yan process $\pi N \rightarrow \gamma_{1}^{*} \gamma_{2}^{*} N^{\prime}$.

Modulo pre-factors, $\Phi^{q}\left(x, \vec{k}_{\perp}^{2}\right)$ is the light-cone wave function of the pion [29, 30]. The double Drell-Yan process implies in both Eq. (2.1) and Eq. (2.3) a staple-like past-pointing Wilson line [31], identical to the one that appears in TMD factorization of the ordinary Drell-Yan process $[32,33,34]$.

\section{Double Drell-Yan process and polarization observables}

To calculate observables we consider the production of two virtual photons rather than two di-lepton pairs. Specifically, we study the process

$$
\pi\left(p_{b}\right)+N\left(p_{a}, \lambda_{a}\right) \rightarrow \gamma_{1}^{*}\left(q_{1}, \lambda_{1}\right)+\gamma_{2}^{*}\left(q_{2}, \lambda_{2}\right)+N^{\prime}\left(p_{a}^{\prime}, \lambda_{a}^{\prime}\right) .
$$

From here on the variables of the incoming and outgoing nucleon carry an index $a$ compared to above. We concentrate on large $s=\left(p_{a}+p_{b}\right)^{2} \approx 2 p_{a}^{+} p_{b}^{-}$, large photon virtualities $q_{1}^{2}, q_{2}^{2}$, and small transverse photon momenta, $\left|\vec{q}_{i \perp}^{2}\right| \ll q_{i}^{2}$. In this region one can use TMD-type factorization. The longitudinal momentum transfer to the nucleon can be written as $\xi_{a}=\left(q_{1}^{+}+q_{2}^{+}\right) /\left(2 P_{a}^{+}\right)$. The LO diagrams for this process are shown in Fig. 1. The scattering amplitude depends on the helicities of the nucleons and photons,

$$
\mathscr{T}_{\lambda_{a}, \lambda_{a}^{\prime}}^{\lambda_{1}, \lambda_{2}}=\mathscr{T}_{\lambda_{a}, \lambda_{a}^{\prime}}^{\mu v} \varepsilon_{\mu}^{*}\left(\lambda_{1}\right) \varepsilon_{v}^{*}\left(\lambda_{2}\right)
$$

where $\varepsilon^{\mu}\left(\lambda_{1}\right)$ and $\varepsilon^{\mu}\left(\lambda_{2}\right)$ are the photon polarization vectors. One finds [25]

$$
\begin{aligned}
\mathscr{T}_{\lambda_{a}, \lambda_{a}^{\prime}}^{\mu \nu}= & i \sum_{q, q^{\prime}} e_{q} e_{q}^{\prime} e^{2} \frac{1}{N_{c}} \int d^{2} \vec{k}_{a \perp} \int d^{2} \vec{k}_{b \perp} \delta^{(2)}\left(\frac{\Delta \vec{q}_{\perp}}{2}-\vec{k}_{a \perp}-\vec{k}_{b \perp}\right) \Phi_{\pi}^{q^{\prime} q}\left(x_{b}, \vec{k}_{b \perp}^{2}\right) \\
& {\left[-i \varepsilon_{\perp}^{\mu \nu}\left(W_{\lambda_{a}, \lambda_{a}^{\prime}}^{q q^{\prime}\left[\gamma^{+}\right]}\left(x_{a}, \vec{k}_{a \perp}\right)-W_{\lambda_{a}, \lambda_{a}^{\prime}}^{q q^{\prime}\left[\gamma^{+}\right]}\left(-x_{a},-\vec{k}_{a \perp}\right)\right)\right.} \\
& \left.-g_{\perp}^{\mu v}\left(W_{\lambda_{a}, \lambda_{a}^{\prime}}^{q q^{\prime}\left[\gamma^{+} \gamma_{5}\right]}\left(x_{a}, \vec{k}_{a \perp}\right)+W_{\lambda_{a}, \lambda_{a}^{\prime}}^{q q^{\prime}\left[\gamma_{5}^{+} \gamma_{5}\right]}\left(-x_{a},-\vec{k}_{a \perp}\right)\right)\right],
\end{aligned}
$$

where $e_{q}$ and $e_{q}^{\prime}$ are the quark charges in units of the elementary charge $e$, and $N_{c}$ is the number of quark colors. The expression in (3.3) describes the double Drell-Yan process for all possible pion and nucleon charge states. Note that $\Phi_{\pi}^{q^{\prime} q}$ is defined as in (2.3), but with the operator $\bar{q}^{\prime} \gamma^{-} \gamma_{5} q$. Isospin symmetry provides $\Phi_{\pi^{+}}^{d u}=\Phi_{\pi^{-}}^{u d}=\sqrt{2} \Phi_{\pi^{0}}^{u u}=-\sqrt{2} \Phi_{\pi^{0}}^{d d}$. Likewise, $W^{q q^{\prime}[\Gamma]}$ is given by (2.1) 
with the operator $\bar{q} \Gamma q^{\prime}$. With this notation one can also describe transitions between different nucleons. Like in the case of transition GPDs, for the GTMDs one has $X_{p \rightarrow n}^{d u}=X_{n \rightarrow p}^{u d}=X_{p}^{u}-X_{p}^{d}$ [35]. In Eq. (3.3) we use the vector $\Delta \vec{q}_{\perp}=\vec{q}_{1 \perp}-\vec{q}_{2 \perp}$. The transverse momenta of the photons can be expressed by $\Delta \vec{q}_{\perp}$ and the transverse momentum transfer to the nucleon $\vec{\Delta}_{a \perp}=-\left(\vec{q}_{1 \perp}+\vec{q}_{2 \perp}\right)$. While the amplitude contains an integration upon the transverse momenta of the quarks, their longitudinal momenta are fixed according to $x_{a}=\left(q_{1}^{+}-q_{2}^{+}\right) /\left(2 P_{a}^{+}\right), x_{b}=1-q_{1}^{-} / p_{b}^{-}=q_{2}^{-} / p_{b}^{-}$. The value for $x_{a}$ implies the so-called ERBL region [36, 37], characterized by $-\xi_{a} \leq x_{a} \leq \xi_{a}$, in which the GTMD matrix element describes the emission of a quark-antiquark pair from the nucleon. The amplitude, a priori, depends on both the $F_{1, i}$ and the $G_{1, i}(i=1, \ldots, 4)$. From (3.3) one readily sees that the dominant contribution to the amplitude is for transversely polarized photons. (Note that $g_{\perp}^{\mu v}=g^{\mu v}-n_{a}^{\mu} n_{b}^{v}-n_{a}^{v} n_{b}^{\mu}$, with the light-like vectors $n_{a}=(1,0,0,-1) / \sqrt{2}, n_{b}=(1,0,0,1) / \sqrt{2}$. $)$

The relation between the scattering amplitude in (3.2) and the cross section in the center-ofmass frame reads [25]

$$
d \sigma_{\lambda_{a}, \lambda_{a}^{\prime}}^{\lambda_{1}, \lambda_{2}}=\frac{\pi}{2 s^{3 / 2}} \frac{1+\xi_{a}}{1-\xi_{a}}\left|\mathscr{T}_{\lambda_{a}, \lambda_{a}^{\prime}}^{\lambda_{1}, \lambda_{2}}\right|^{2} \delta\left(p_{a}^{\prime 0}+q_{1}^{0}+q_{2}^{0}-\sqrt{s}\right) \frac{d^{4} q_{1}}{(2 \pi)^{4}} \frac{d^{4} q_{2}}{(2 \pi)^{4}}
$$

where we have already integrated over the phase space of the outgoing nucleon. Below we consider the unpolarized cross section, single-spin asymmetries (SSAs), and double-spin asymmetries (DSAs). For this purpose it is convenient to introduce

$$
\begin{aligned}
\tau_{U U} & =\frac{1}{2} \sum_{\lambda, \lambda^{\prime}}\left|\mathscr{T}_{\lambda, \lambda^{\prime}}\right|^{2}, \\
\tau_{L U} & =\frac{1}{2} \sum_{\lambda^{\prime}}\left(\left|\mathscr{T}_{+, \lambda^{\prime}}\right|^{2}-\left|\mathscr{T}_{-, \lambda^{\prime}}\right|^{2}\right), \\
\tau_{L L} & =\frac{1}{2}\left(\left(\left|\mathscr{T}_{+,+}\right|^{2}-\left|\mathscr{T}_{+,-}\right|^{2}\right)-\left(\left|\mathscr{T}_{-,+}\right|^{2}-\left|\mathscr{T}_{-,-}\right|^{2}\right)\right),
\end{aligned}
$$

where summation over the photon polarizations is implied. Obviously, $\tau_{L U}$ determines the numerator of the longitudinal target SSA, whereas $\tau_{L L}$ describes the longitudinal DSA with polarization of both the target and the recoil nucleon. Spin asymmetries for transverse polarization in the (transverse) $x$-direction or $y$-direction are defined accordingly.

In order to get direct access to $F_{1,4}$, that is, without interference with other GTMDs, one has to consider a linear combination of (polarization) observables [25],

$$
\begin{aligned}
\frac{1}{4}\left(\tau_{U U}+\tau_{L L}-\tau_{X X}-\tau_{Y Y}\right)= & \frac{2}{M^{4}}\left(\varepsilon_{\perp}^{i j} \Delta q_{\perp}^{i} \Delta_{a \perp}^{j}\right)^{2} C^{(+)}\left[\vec{\beta}_{\perp} \cdot \vec{k}_{a \perp} F_{1,4} \Phi_{\pi}\right] C^{(+)}\left[\vec{\beta}_{\perp} \cdot \vec{k}_{a \perp} F_{1,4}^{*} \Phi_{\pi}^{*}\right] \\
& +2 C^{(+)}\left[G_{1,4} \Phi_{\pi}\right] C^{(+)}\left[G_{1,4}^{*} \Phi_{\pi}^{*}\right] .
\end{aligned}
$$

In Eq. (3.8) we use the shorthand notation

$$
\begin{aligned}
C^{( \pm)}\left[w\left(\vec{k}_{a \perp}, \vec{k}_{b \perp}\right) X \Phi_{\pi}\right]= & \frac{e^{2}}{\sqrt{1-\xi_{a}^{2}} N_{c}} \sum_{q, q^{\prime}} e_{q} e_{q}^{\prime} \int d^{2} \vec{k}_{a \perp} \int d^{2} \vec{k}_{b \perp} \delta^{(2)}\left(\frac{\Delta \vec{q}_{\perp}}{2}-\vec{k}_{a \perp}-\vec{k}_{b \perp}\right) \\
& \times w\left(\vec{k}_{a \perp}, \vec{k}_{b \perp}\right)\left[X^{q q^{\prime}}\left(x_{a}, \vec{k}_{a \perp}\right) \pm X^{q q^{\prime}}\left(-x_{a},-\vec{k}_{a \perp}\right)\right] \Phi_{\pi}^{q^{\prime} q}\left(x_{b}, \vec{k}_{b \perp}^{2}\right)
\end{aligned}
$$


with $w\left(\vec{k}_{a \perp}, \vec{k}_{b \perp}\right)$ a generic weight function. The vector $\vec{\beta}_{\perp}$ in (3.8) reads

$$
\vec{\beta}_{\perp}=\frac{\vec{\Delta}_{a \perp}^{2} \Delta \vec{q}_{\perp}-\left(\vec{\Delta}_{a \perp} \cdot \Delta \vec{q}_{\perp}\right) \vec{\Delta}_{a \perp}}{\vec{\Delta}_{a \perp}^{2} \Delta \vec{q}_{\perp}^{2}-\left(\vec{\Delta}_{a \perp} \cdot \Delta \vec{q}_{\perp}\right)^{2}} .
$$

We repeat that in order to obtain Eq. (3.8) the photon polarizations have been summed over. While in that case there is no interference between $F_{1,4}$ and other GTMDs, one still has a second term which is given by $G_{1,4}$. As already mentioned, $G_{1,4}$ is presumably large, and therefore it may actually be difficult to address $F_{1,4}$ through this observable, unless one has a reliable estimate of $G_{1,4}$. However, one can separate the two contributions in (3.8) by not summing over the photon polarizations. For instance, if one projects on appropriate linear polarizations of the photons, the contributions of either $F_{1,4}$ or $G_{1,4}$ can be switched off [31]. This result, which holds irrespective of the polarization states of the nucleons, follows from the expression in (3.3). To address $G_{1,1}$ one can study $\frac{1}{4}\left(\tau_{U U}+\tau_{L L}+\tau_{X X}+\tau_{Y Y}\right)$ [25]. The result for this linear combination is identical to (3.8), but with the replacements $F_{1,4} \rightarrow G_{1,1}$ and $G_{1,4} \rightarrow F_{1,1}$. Again, the contributions from $G_{1,1}$ and $F_{1,1}$ can be separated by measuring suitable photon polarizations.

Apart from the fact that a considerable number of different polarization measurements is required, the observable in (3.8), as well as the corresponding observable for $G_{1,1}$, may have a drawback: In these linear combinations one has cancellations of potentially large terms [31]. Specifically, for instance the individual polarization observables entering (3.8) have terms proportional to $F_{1,1} F_{1,1}^{*}$, which can be expected to be large (see the discussion in the paragraph after Eq. (2.2)). It may therefore be beneficial to also explore interference between $F_{1,4}$ (or $G_{1,1}$ ) and other GTMDs. Such an interference shows up in the following linear combination of longitudinal SSAs [25]:

$$
\begin{aligned}
\frac{1}{2}\left(\tau_{L U}+\tau_{U L}\right)= & \frac{1}{2}\left(\left|\mathscr{T}_{+,+}\right|^{2}-\left|\mathscr{T}_{-,-}\right|^{2}\right) \\
=\frac{4}{M^{2}} \varepsilon_{\perp}^{i j} \Delta q_{\perp}^{i} \Delta_{a \perp}^{j} \operatorname{Im} & \left\{C^{(-)}\left[F_{1,1} \Phi_{\pi}\right] C^{(+)}\left[\vec{\beta}_{\perp} \cdot \vec{k}_{a \perp} F_{1,4}^{*} \Phi_{\pi}^{*}\right]\right. \\
& \left.-C^{(+)}\left[G_{1,4} \Phi_{\pi}\right] C^{(-)}\left[\vec{\beta}_{\perp} \cdot \vec{k}_{a \perp} G_{1,1}^{*} \Phi_{\pi}^{*}\right]\right\}
\end{aligned}
$$

We point out that the expressions for $\tau_{L U}$ or $\tau_{U L}$ alone are more complicated as they contain additional GTMDs [31]. More polarization observables exist which involve interference between $F_{1,4}$ (or $G_{1,1}$ ) and other GTMDs, but the observable in (3.11) gives the simplest expression [31]. Note that on the r.h.s. of (3.11) the imaginary part of products of GTMDs appears. According to current knowledge the GTMDs most relevant for the spin structure of the nucleon are $\operatorname{Re} F_{1,4}$ and $\operatorname{Re} G_{1,1}$. Though these functions contribute to (3.11) they interfere with $\operatorname{Im} F_{1,4}$ and $\operatorname{Im} G_{1,1}$, respectively. At present, there exists no information on the latter functions, and they may in fact be small. This issue can be overcome by considering the observable $\frac{1}{2}\left(\tau_{X Y}-\tau_{Y X}\right)$, whose result agrees with the r.h.s. of (3.11) but with $\operatorname{Re}\{\ldots\}$ instead of $\operatorname{Im}\{\ldots\}$. Finally, we repeat that $F_{1,1}$ (and $G_{1,4}$ ) are presumably large. Therefore, a term of the form $F_{1,1} F_{1,4}^{*}$ can be expected to be larger than the term $F_{1,4} F_{1,4}^{*}$ which appears in Eq. (3.8). Presently available model calculations for GTMDs do not allow one to quantify this statement in the kinematical (ERBL) region of interest. 


\section{Summary and Outlook}

We have shown that GTMDs for quarks can be studied through the exclusive double DrellYan process [25]. Specifically, to leading order in perturbative QCD, this process in sensitive to GTMDs in the ERBL region. The main focus was on the GTMDs $F_{1,4}$ and $G_{1,1}$ which recently attracted much attention because of their relation to the spin structure of the nucleon. The double Drell-Yan process leads to a staple-like Wilson line for the operator definition of the GTMDs [31], providing the connnection to the canonical OAM $\left(L_{\mathrm{JM}}\right)$ [12]. We have proposed several polarization observables which can give access to these GTMDs, either directly or through interference with other GTMDs. In a similar manner, other leading-twist GTMDs could be explored via suitable polarization observables [31].

Several extensions of our work can be envisioned. An attempt should be made to numerically estimate both the unpolarized cross section and the various spin asymmetries to find out if the reaction $\pi N \rightarrow\left(\ell_{1}^{-} \ell_{1}^{+}\right)\left(\ell_{2}^{-} \ell_{2}^{+}\right) N^{\prime}$ is measurable at existing facilities. We repeat that the exclusive double Drell-Yan reaction is the first known exclusive process involving the nucleon which is directly sensitive to transverse quark momenta. It therefore holds promise to give experimental access to the so far elusive GTMDs for quarks. We also note that one can perform a similar analysis for nucleon-nucleon collisions [31]. Production of heavy gauge bosons instead of photons may be considered as well. Moreover, hadronic final states typically give rise to higher count rates. One such example is the process $p p \rightarrow \eta_{c} \eta_{c} p p$, which can basically be treated along the lines discussed above, though gluon GTMDs enter the leading-order analysis [31]. One might expect that arguments similar to the ones used to justify factorization for inclusive double-parton scattering (see, e.g., Refs. [38, 39, 40]) apply to these proposed processes. We finally point out that the type of reactions discussed here could also constrain GPDs in the ERBL region, where experimental information is still sparse - see Refs. [41, 42, 43] for related work on GPDs.

\section{Acknowledgments}

This work has been supported by the National Science Foundation of the United States under Contract No. PHY-1516088 (S.B., A.M.), the National Science Foundation of China under Grant No. 11675093, and by the Thousand Talents Plan for Young Professionals (J.Z.).

\section{References}

[1] D. Boer et al., Gluons and the quark sea at high energies: distributions, polarization, tomography, arXiv:1108.1713 [nucl-th].

[2] A. Accardi et al., Electron ion collider: the next QCD frontier: understanding the glue that binds us all, Eur. Phys. J. A 52, 268 (2016) [arXiv:1212.1701 [nucl-ex]].

[3] S. Meissner, A. Metz, M. Schlegel and K. Goeke, Generalized parton correlation functions for a spin-0 hadron, JHEP 0808, 038 (2008) [arXiv:0805.3165 [hep-ph]].

[4] S. Meissner, A. Metz and M. Schlegel, Generalized parton correlation functions for a spin-1/2 hadron, JHEP 0908, 056 (2009) [arXiv:0906.5323 [hep-ph]]. 
[5] C. Lorcé and B. Pasquini, Structure analysis of the generalized correlator of quark and gluon for a spin-1/2 target, JHEP 1309, 138 (2013) [arXiv:1307.4497 [hep-ph]].

[6] X.-D. Ji, Viewing the proton through 'color' filters, Phys. Rev. Lett. 91, 062001 (2003) [hep-ph/0304037].

[7] A. V. Belitsky, X.-D. Ji and F. Yuan, Quark imaging in the proton via quantum phase space distributions, Phys. Rev. D 69, 074014 (2004) [hep-ph/0307383].

[8] C. Lorcé, B. Pasquini and M. Vanderhaeghen, Unified framework for generalized and transverse-momentum dependent parton distributions within a $3 Q$ light-cone picture of the nucleon, JHEP 1105, 041 (2011) [arXiv:1102.4704 [hep-ph]].

[9] C. Lorcé and B. Pasquini, Quark Wigner distributions and orbital angular momentum, Phys. Rev. D 84, 014015 (2011) [arXiv:1106.0139 [hep-ph]].

[10] C. Lorcé, Spin-orbit correlations in the nucleon, Phys. Lett. B 735, 344 (2014) [arXiv:1401.7784 [hep-ph]].

[11] K. Kanazawa, C. Lorcé A. Metz, B. Pasquini and M. Schlegel, Twist-2 generalized transverse-momentum dependent parton distributions and the spin/orbital structure of the nucleon, Phys. Rev. D 90, 014028 (2014) [arXiv:1403.5226 [hep-ph]].

[12] Y. Hatta, Notes on the orbital angular momentum of quarks in the nucleon, Phys. Lett. B 708, 186 (2012) [arXiv:1111.3547 [hep-ph]].

[13] X.-D. Ji, X. Xiong and F. Yuan, Proton spin structure from measurable parton distributions, Phys. Rev. Lett. 109, 152005 (2012) [arXiv:1202.2843 [hep-ph]].

[14] R. L. Jaffe and A. Manohar, The G(1) problem: fact and fantasy on the spin of the proton, Nucl. Phys. B 337, 509 (1990).

[15] X.-D. Ji, Gauge-invariant decomposition of nucleon spin, Phys. Rev. Lett. 78, 610 (1997) [hep-ph/9603249].

[16] M. Burkardt, Parton orbital angular momentum and final state interactions, Phys. Rev. D 88, 014014 (2013) [arXiv:1205.2916 [hep-ph]].

[17] M. Engelhardt, Quark orbital dynamics in the proton from Lattice QCD - from Ji to Jaffe-Manohar orbital angular momentum, arXiv:1701.01536 [hep-lat].

[18] A. Rajan, A. Courtoy, M. Engelhardt and S. Liuti, Parton transverse momentum and orbital angular momentum distributions, Phys. Rev. D 94, 034041 (2016) [arXiv:1601.06117 [hep-ph]].

[19] Y. Hatta, B. W. Xiao and F. Yuan, Probing the small-x gluon tomography in correlated hard diffractive dijet production in deep inelastic scattering, Phys. Rev. Lett. 116, 202301 (2016) [arXiv:1601.01585 [hep-ph]].

[20] Y. Hatta, Y. Nakagawa, F. Yuan, Y. Zhao and B. Xiao, Gluon orbital angular momentum at small-x, Phys. Rev. D 95, 114032 (2017) [arXiv:1612.02445 [hep-ph]].

[21] X. Ji, F. Yuan and Y. Zhao, Hunting the gluon orbital angular momentum at the Electron-Ion Collider, Phys. Rev. Lett. 118, 192004 (2017) [arXiv:1612.02438 [hep-ph]].

[22] J. Zhou, Elliptic gluon generalized transverse-momentum-dependent distribution inside a large nucleus, Phys. Rev. D 94, 114017 (2016) [arXiv:1611.02397 [hep-ph]].

[23] Y. Hagiwara, Y. Hatta and T. Ueda, Wigner, Husimi and GTMD distributions in the Color Glass Condensate, Phys. Rev. D 94, 094036 (2016) [arXiv:1609.05773 [hep-ph]]. 
[24] Y. Hagiwara, Y. Hatta, B. W. Xiao and F. Yuan, Elliptic flow in small systems due to elliptic gluon distributions?, Phys. Lett. B 771, 374 (2017) [arXiv:1701.04254 [hep-ph]].

[25] S. Bhattacharya, A. Metz and J. Zhou, Generalized TMDs and the exclusive double Drell-Yan process, Phys. Lett. B 771, 396 (2017) [arXiv:1702.04387 [hep-ph]].

[26] J. Collins, Foundations of perturbative $Q C D$, Cambridge monographs on particle physics, nuclear physics and cosmology, 32.

[27] M. G. Echevarria, A. Idilbi, K. Kanazawa, C. Lorcé, A. Metz, B. Pasquini and M. Schlegel, Proper definition and evolution of generalized transverse momentum dependent distributions, Phys. Lett. B 759, 336 (2016) [arXiv:1602.06953 [hep-ph]].

[28] D. E. Soper, Infinite-momentum helicity states, Phys. Rev. D 5, 1956 (1972).

[29] M. Diehl, Generalized parton distributions, Phys. Rept. 388, 41 (2003) [hep-ph/0307382].

[30] S. J. Brodsky, H. C. Pauli and S. S. Pinsky, Quantum chromodynamics and other field theories on the light cone, Phys. Rept. 301, 299 (1998) [hep-ph/9705477].

[31] S. Bhattacharya, A. Metz, J. Zhou, Generalized TMDs in the exclusive pion-nucleon and nucleon-nucleon double Drell-Yan process, in preparation.

[32] J. C. Collins, Leading twist single transverse-spin asymmetries: Drell-Yan and deep inelastic scattering, Phys. Lett. B 536, 43 (2002) [hep-ph/0204004].

[33] X.-D. Ji and F. Yuan, Parton distributions in light cone gauge: where are the final state interactions?, Phys. Lett. B 543, 66 (2002) [hep-ph/0206057].

[34] A. V. Belitsky, X.-D. Ji and F. Yuan, Final state interactions and gauge invariant parton distributions, Nucl. Phys. B 656, 165 (2003) [hep-ph/0208038].

[35] L. Mankiewicz, G. Piller and T. Weigl, Hard leptoproduction of charged vector mesons, Phys. Rev. D 59, 017501 (1999) [hep-ph/9712508].

[36] A. V. Efremov and A. V. Radyushkin, Factorization and asymptotical behavior of pion form factor in QCD, Phys. Lett. 94B, 245 (1980).

[37] G. P. Lepage and S. J. Brodsky, Exclusive processes in quantum chromodynamics: evolution equations for hadronic wave functions and the form factors of mesons, Phys. Lett. 87B, 359 (1979).

[38] M. Diehl and A. Schäfer, Theoretical considerations on multiparton interactions in QCD, Phys. Lett. B 698, 389 (2011) [arXiv:1102.3081 [hep-ph]].

[39] M. Diehl, D. Ostermeier and A. Schäfer, Elements of a theory for multiparton interactions in QCD, JHEP 1203, 089 (2012) Erratum: [JHEP 1603, 001 (2016)] [arXiv:1111.0910 [hep-ph]].

[40] M. Diehl, J. R. Gaunt, D. Ostermeier, P. Plößl and A. Schäfer, Cancellation of Glauber gluon exchange in the double Drell-Yan process, JHEP 1601, 076 (2016) [arXiv:1510.08696 [hep-ph]].

[41] E. R. Berger, M. Diehl and B. Pire, Probing generalized parton distributions in $\pi N \rightarrow \ell^{+} \ell^{-} N$, Phys. Lett. B 523, 265 (2001) [hep-ph/0110080].

[42] S. V. Goloskokov and P. Kroll, The exclusive limit of the pion-induced Drell-Yan process, Phys. Lett. B 748, 323 (2015) [arXiv:1506.04619 [hep-ph]].

[43] T. Sawada, W. C. Chang, S. Kumano, J. C. Peng, S. Sawada and K. Tanaka, Accessing proton generalized parton distributions and pion distribution amplitudes with the exclusive pion-induced Drell-Yan process at J-PARC, Phys. Rev. D 93, 114034 (2016) [arXiv:1605.00364 [nucl-ex]]. 